\title{
Multisession Gamma Knife surgery for benign orbital tumors
}

\author{
Clinical article
}

\author{
Kyung-Il Jo, M.D., ${ }^{1}$ Yong Seok Im, Ph.D., ${ }^{1}$ Doo-Sik Kong, M.D., Ph.D., ${ }^{1}$ \\ Ho Jun Seol, M.D., Ph.D., ${ }^{1}$ Do-Hyun Nam, M.D., Ph.D., ${ }^{1}$ Yoon-Duck Kim, M.D., Ph.D.,2 \\ And Jung-IL Lee, M.D., Ph.D. ${ }^{1}$ \\ Departments of ${ }^{1}$ Neurosurgery and ${ }^{2}$ Ophthalmology, Samsung Medical Center, Sungkyunkwan University \\ School of Medicine, Seoul, Korea
}

Object. The goal of this study was to investigate the safety and efficacy of multisession Gamma Knife surgery (GKS) in the treatment of benign orbital tumors.

Methods. Twenty-three patients who retained their vision despite having tumors touching their optic nerve were treated with multisession (4-fraction) GKS. The median tumor volume was $2800 \mathrm{~mm}^{3}$ (range 211-10,800 $\mathrm{mm}^{3}$ ), and the median cumulative margin dose was 20 Gy (range 18-22 Gy).

Results. The median clinical follow-up duration in these patients was 38 months (range 9-74 months). No patient experienced tumor progression in this study. In particular, a higher degree of tumor shrinkage was found in the 7 patients with cavernous hemangiomas than in patients with other types of lesions $(p<0.05)$. Of the 23 patients whose preoperative vision was preserved, 11 showed improvement in visual acuity and/or visual field and 12 showed stable visual acuity. No GKS-related adverse events were noted during or after treatment.

Conclusions. Multisession radiosurgery using the Gamma Knife may be a good strategy for tumors in direct contact with the optic nerve. A cumulative margin dose of up to $22 \mathrm{~Gy}$ delivered in 4 sessions is safe for preservation of visual function with a high probability of tumor control.

(http://thejns.org/doi/abs/10.3171/2012.7.GKS12780)

\section{KeY Words • Gamma Knife surgery • orbital tumor • multisession radiosurgery $\bullet \quad$ stereotactic radiosurgery}

$\mathrm{T}$ HE orbit is a pyramidal space that contains the eyeball, extraocular muscles, vessels, and nerves. Since all anatomical structures of the orbit can give rise to a neoplasm, the heterogeneous array of potential lesions that can develop in this region poses numerous challenges to diagnosis, imaging, and management. , $^{5,811}$ Common symptoms are visual disturbance and proptosis. Visual loss associated with orbital tumors may result from direct compression of the optic nerve or circulatory impairment of the vasculature leading to the optic apparatus. The goal of treatment is tumor control without vision deterioration; however, the optimal management remains controversial. Currently, primary radiotherapy and surgery followed by radiotherapy are common treatment modalities for orbital tumors, although inadequate tumor control and subsequent vision deterioration are often reported. 6,9

Recent articles have reported that SRS is a good option for the treatment of intraorbital lesions. ${ }^{1,11,19}$ In this study, we evaluated the safety and effectiveness of fractionated GKS as a primary or secondary treatment for benign orbital tumors.

\footnotetext{
Abbreviations used in this paper: GKS = Gamma Knife surgery; $\mathrm{SRS}=$ stereotactic radiosurgery.
}

\section{Methods}

\section{Patient Population}

Between April 2004 and December 2010, 46 patients with orbital tumors underwent GKS at our center. Nine patients were excluded because they had malignant tumors or had received fractionated external beam radiation treatment or concomitant local or systemic chemotherapy. Twenty-three of the remaining 37 patients underwent multisession (4-session) GKS for benign orbital tumors. Single-session GKS was performed for intraocular lesions such as choroidal hemangioma or patients who were already blind. Patients who had lesions close to the optic apparatus and retained vision were treated with multisession radiosurgery. The 23 patients who underwent multisession GKS were included in the study popuation. This study was approved by the institutional review board of our hospital.

Among the 23 patients treated with multisession GKS, there were 15 women $(65.2 \%)$ and 8 men (34.8\%), with a mean age of $41.5 \pm 12.3$ years. No patient had a history of previous standard radiotherapy. Eight patients $(34.8 \%)$ had previously undergone open resection (craniotomy or the Krönlein operation) or fine-needle aspiration biopsy. The diagnoses were presumed based on characteristic clinical and neuroimaging findings in the other 
15 patients (Table 1). The most common lesions were meningioma ( 9 patients) and cavernous hemangioma (9 patients). Other benign lesions encountered in our case series were schwannoma (3 patients), solitary fibrous tumor (1 patient), and granular cell tumor (1 patient) of the orbit. The patients' clinical symptoms and signs are listed in Table 2. The most common clinical symptom was visual disturbance.

Tumors are classified as located in the orbital apex, when placed posterior to a plane passing through the greater wings of the sphenoid bone, at the level of the anterior border of the middle cranial fossa. Classification on a coronal plane anterior to the orbital apex was defined by $180^{\circ}$-angled sectors by vertical lines intersecting in correspondence to the optic nerve.

\section{Treatment Protocol}

After a local anesthetic had been applied to the head, a Leksell model $G$ stereotactic frame (Elekta $A B$ ) was affixed to the head, and MR imaging was performed for target localization and planning purposes. Axial T2weighted images with a slice thickness of $2 \mathrm{~mm}$ and 3D spoiled-gradient recalled images with double-dose contrast enhancement were acquired at a slice thickness of 1 $\mathrm{mm}$ with no slice interval. The fat suppression technique was used to improve delineation of the optic nerve. Images were transferred to a workstation, and radiosurgical planning was generated using Leksell GammaPlan software (versions 5.34 and 9.0, Elekta AB). Target volumes and critical structures were delineated manually by the treating surgeon on axial images with simultaneous overlay of the outlines on coronal and sagittal images. Until June 2010, radiosurgical treatment was performed using the Leksell Gamma Knife model C (Elekta AB); subsequently, GKS was performed using the Perfexion model. The dose-planning method used for multisession radiosurgery was similar to that used for single-session radiosurgery, except for the prescribed dose, which was adjusted on the basis of the dose directed to the optic apparatus and the number of fractions used. All patients were treated with multisession radiosurgery in 4 fractions with 12-hour intervals. The median cumulative margin dose was 20 Gy (18-22 Gy) at the $50 \%$ isodose line. The accuracy of stereotaxy was checked before the last session by performing MR imaging, and the mean interfractional displacement error was found to be $0.19 \mathrm{~mm}$ (range $0-0.56 \mathrm{~mm}$ ). The stereotactic frame was removed at the end of the treatment, and the patient was discharged on the same day or on the following day.

\section{Follow-Up Examinations}

Patients underwent follow-up examinations that were conducted by the neurosurgeon and an ophthalmologist. Visual acuity and field examination, clinical evaluation, and brain MR images were obtained at 3-6 months and 1 year after GKS; thereafter, follow-up examinations were performed annually. The response to treatment was defined clinically by an improvement in vision or symptoms in comparison to the patient's status before GKS. The response was determined using regular visual field (perimetry) tests, best corrected visual acuity measure-
TABLE 1: Characteristics in 23 patients treated with multisession GKS

\begin{tabular}{lc}
\hline \multicolumn{1}{c}{ Characteristic } & Value \\
\hline no. of patients & 23 \\
mean age (yrs) & $41.5 \pm 12.3$ \\
male/female ratio & $8: 15$ \\
median tumor vol in $\mathrm{mm}^{3}$ (range) & \\
tumor type & $2,800(221-10,800)$ \\
$\quad$ meningioma & 9 \\
cavernous hemangioma & 9 \\
schwannoma & 3 \\
granular cell tumor & 1 \\
$\quad$ solitary fibrous tumor & 1 \\
previous treatment (8 patients) & \\
partial removal & 4 \\
biopsy & 4 \\
median cumulative marginal dose in Gy (range) & $20(18-22)$ \\
mean interfractional error in mm (range) & $0.19(0-0.56)$ \\
location of tumor & \\
medial & 7 \\
lateral & 3 \\
apex & 13 \\
\hline
\end{tabular}

ments (made using the Snellen chart), and volumetry based on MR images of the orbit. Subjective values such as "counting fingers," "hand movements," "light perception," and "no light perception" were converted into a decimal acuity of $0.01,0.005,0.001$, and 0 , respectively. An improvement or deterioration in vision was defined as a gain or loss of more than 2 lines on the Snellen chart. Disease progression was defined as visual deterioration or as imaging-verified tumor progression. Tumor volume measurements from each follow-up MR imaging examination were collected, and possible adverse radiation effects were assessed and compared during each MR imaging study. Tumor volumes before and after treatment were estimated using Leksell GammaPlan software based on data from the MR images. The tumor volume response was classified as follows: shrinkage (> 20\% decrease in tumor volume), stable (0\%-20\% change in tumor volume), and tumor progression ( $>20 \%$ increase in tumor volume) at the last follow-up examination. We could not obtain follow-up MR images in 2 patients with cavernous hemangiomas.

TABLE 2: Clinical symptoms and signs in 23 patients treated with multisession GKS

\begin{tabular}{lc}
\hline \multicolumn{1}{c}{ Symptom or Sign } & No. of Patients \\
\hline visual acuity/field change & 16 \\
proptosis & 11 \\
diplopia & 8 \\
headache or orbital pain & 4 \\
\hline
\end{tabular}




\section{Statistical Analysis}

The Mann-Whitney test was used to compare the shrinkage rate between disease groups. A p value $<0.05$ (2-tailed) was considered statistically significant. Data were analyzed using a statistical software package (SPSS version 18.0, SPSS Inc.).

\section{Results}

\section{Imaging Results}

The median imaging follow-up period was 27 months (range 4-72 months; 21 patients). Only clinical follow-up was possible in 2 cases of cavernous hemangioma because the patients refused follow-up imaging. All patients who underwent follow-up imaging examinations experienced either tumor volume shrinkage (11 patients) or stabilization (10 patients) during the follow-up period. Tumor shrinkage proceeded in a concentric fashion, and there were no cystic changes in the tumors.

In the 7 cases of cavernous hemangioma, the median reduction in tumor volume was $57.1 \%$ (range $14.3 \%-91.9 \%$ ), whereas in the 9 cases of meningioma, the median reduction in tumor volume was $12.6 \%$ (range $10.5 \%-51.5 \%$ ) during a similar follow-up period. This difference was statistically significant ( $\mathrm{p}<0.05$, Mann-Whitney U-test; Figs. 1 and 2). Three schwannomas exhibited volume reduction rates of $14 \%, 78 \%$, and $92 \%$ during 5, 27, and 48 months of follow-up, respectively. The tumor volume reduction rate was $43 \%$ after 5 months of follow-up for a solitary fibrous tumor and 55\% after 19 months for a granular cell tumor. No acute or subacute radiosurgery-related adverse event was observed during this study. In 1 case there was a transient increase in tumor volume during the follow-up period; however, tumor shrinkage was evident on subsequent follow-up MR images (Case 2; Fig. 1).

\section{Clinical Results}

The median clinical follow-up period was 38 months (range 9-74 months). Seven of 11 patients with proptosis showed improvement in varying degrees. Orbital pain disappeared in 3 patients and remained stable in 1 patient. Visual acuity remained stable or improved in all patients (Table 3). Seven of the 11 patients who had visual field abnormalities before radiosurgery experienced significant improvements in vision (Fig. 3; Table 3). Changes in visual acuity according to the follow-up time course and the dose of radiation directed to the optic apparatus are detailed in Table 4.

Tumor control was obtained in all 7 patients with a benign orbital tumor that had been treated with singlesession GKS; however, these patients were already blind and their visual status remained unchanged.

\section{Discussion}

The current treatment options for orbital tumors are surgical removal, radiotherapy, and GKS. Although a surgical approach is frequently possible, open surgery is inherently associated with additional case-specific risks. Moreover, attempts at complete resection of tumors in critical locations, such as the optic nerve sheath, optic canal, or superior orbital fissure, may result in direct injury to, or vascular impairment of, the optic apparatus followed by vision loss. ${ }^{20}$ In our case series, a common location of orbital tumors was the medial aspect or orbit apex area. Resection of tumors in these critical and confined spaces remains a challenge, because the visually critical structures are tightly packed and tenaciously adherent to apical structures, so even careful surgical removal can imperil vision. ${ }^{7}$ Thus, surgery should be reserved for select cases, such as patients with severe proptosis or patients in whom there is sufficient space for the optic apparatus. ${ }^{3}$

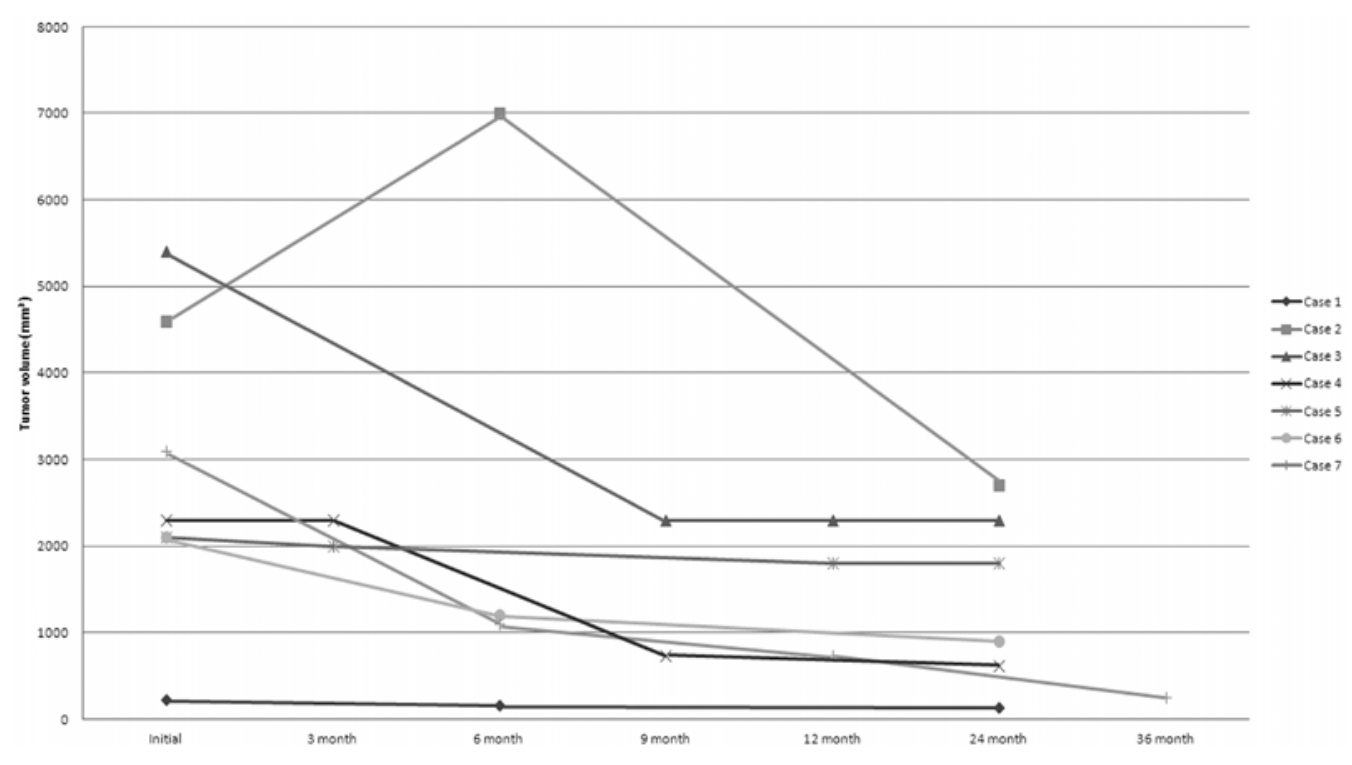

FIG. 1. Line graph showing lesion volume changes over time following multisession SRS in 7 cases of cavernous hemangioma. All but 1 patient (Case 5) had remarkable tumor shrinkage during the follow-up period after fractionated GKS. The radiosurgical response was consistent during follow-up. The median follow-up period was 24 months. 


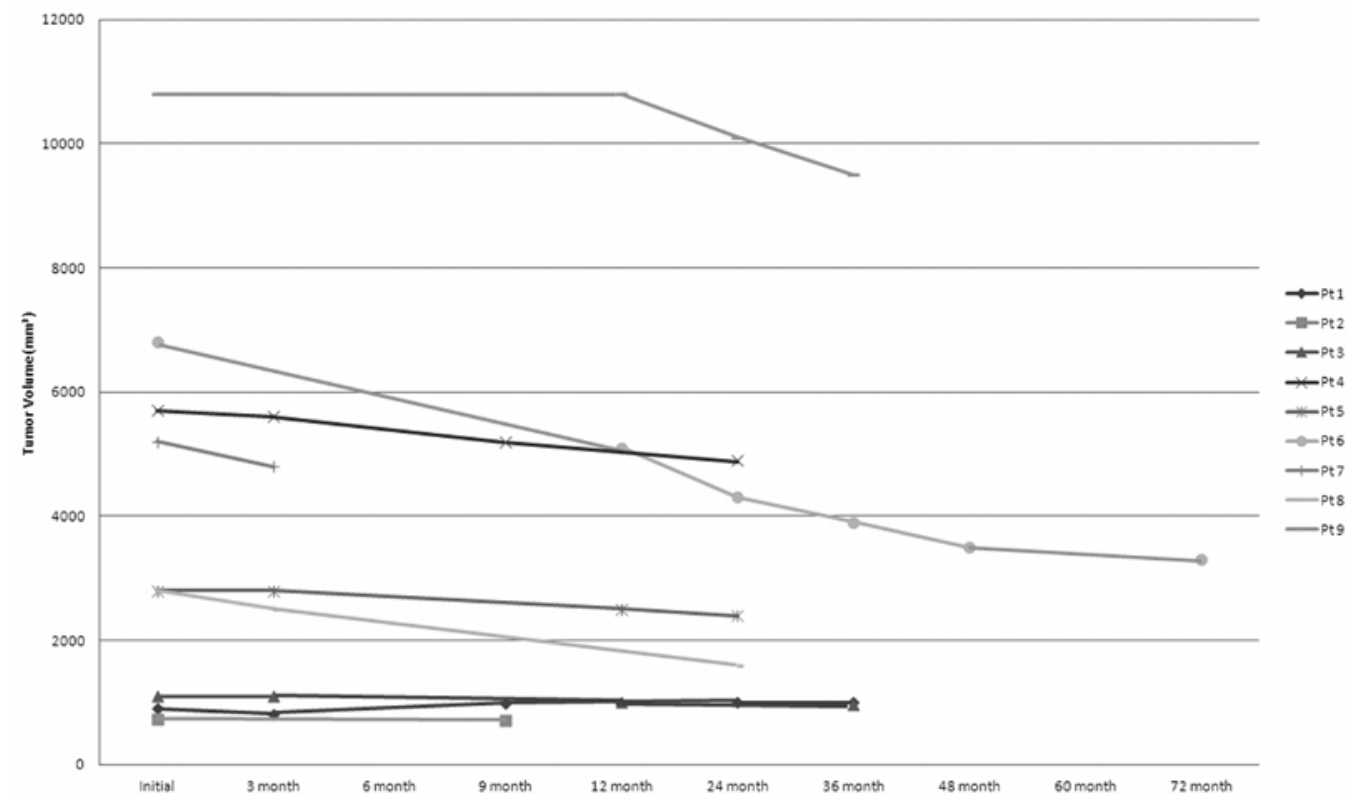

FIG. 2. Line graph showing lesion volume changes over time following multisession SRS in 9 cases of meningioma. Only 2 patients (Cases 6 and 8) showed significant tumor shrinkage during the follow-up period. No cases of tumor progression were identified in this group. $\mathrm{Pt}=$ patient/case.

Consequently, orbital tumors are frequently treated using radiotherapy alone or by performing an initial debulking followed by radiotherapy. Because of spatial inaccuracies in patient setup, standard methods of radiotherapy irradiate a large region of normal brain. Although the short-term side effects of such irradiation seem minor, the long-term consequences are largely unstudied and potentially deleterious. ${ }^{1}$ It is worth emphasizing that optic nerve injury has been reported with even the most sophisticated and accurate of modern conventional fractionated radiotherapy regimens..$^{15}$ Therefore, the radiation dose should be limited to avoid complications, and it is inevitable that tumor control is often unsatisfactory. Currently, radiosurgery is a well-established treatment option for small intracranial lesions of various pathological types. ${ }^{12,17,18}$ In particular, radiosurgery has been shown to produce an effect similar to that of complete resection

\section{TABLE 3: Outcomes in 23 patients treated with multisession GKS}

\begin{tabular}{lc}
\hline \multicolumn{1}{c}{ Variable } & Value \\
\hline median clinical follow-up in mos (range) & $38(9-74)$ \\
median imaging follow-up in mos (range) & $27(4-72)$ \\
visual acuity_no. of patients (\%) & \\
$\quad$ improved & $11(47.8)$ \\
$\quad$ stable & $12(52.2)$ \\
visual field defect (11 patients)_no. of patients (\%) & \\
improved & $7(63.6)$ \\
stable & $4(36.4)$ \\
overall tumor control-no. of patients (\%) & \\
stable & $10(43.5)$ \\
shrinkage & $11(47.8)$ \\
not available & $2(8.7)$ \\
\hline
\end{tabular}

for selected small benign lesions.,.$^{2,12}$ Radiosurgery has the advantage of better spatial accuracy than fractionated radiotherapy, and the biologically equivalent dose of high single-dose radiosurgery could possibly exceed that of
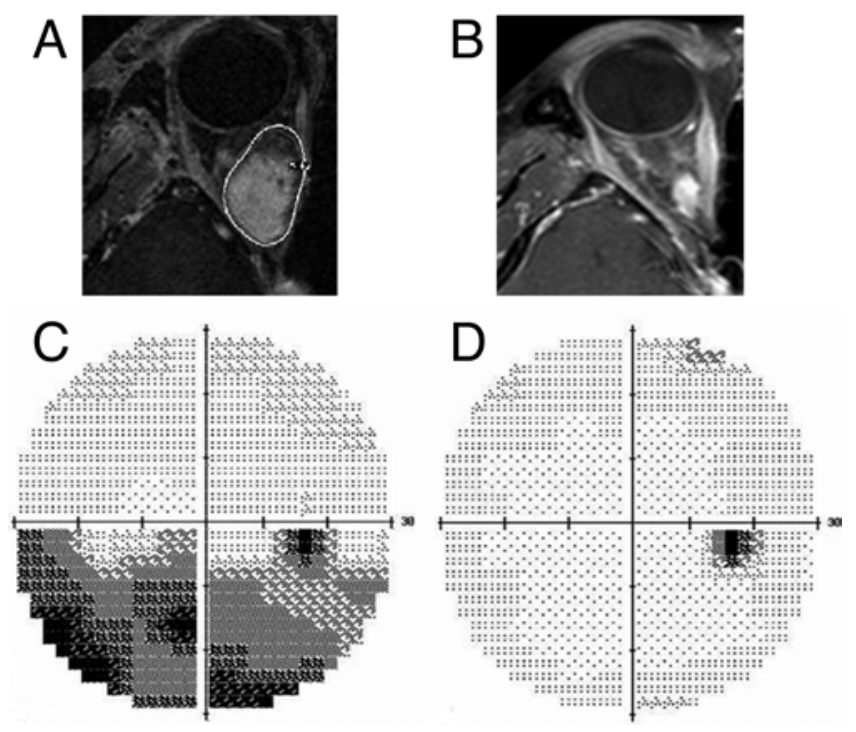

FIG. 3. Case 4. Magnetic resonance images (A and B) and computer-generated visual field images (C and D). This 26-year-old man underwent radiosurgery for a cavernous hemangioma of the right orbit. The pretreatment MR image $(A)$ shows a cavernous hemangioma involving the right orbital apex area. The patient underwent fractionated GKS with a total dose of 20 Gy to the tumor margin delivered in 4 stages. An MR image (B) obtained 24 months after GKS demonstrates marked volume reduction of the mass. A visual field examination of the affected eye was obtained prior to GKS (C). A follow-up visual field examination 24 months after treatment (D) showed improvement in the patient's visual field defect. The man's best corrected visual acuity increased from 0.6 to 1.0 , and his exophthalmos improved. 
K. I. Jo et al.

TABLE 4: Visual acuity changes after multisession GKS

\begin{tabular}{|c|c|c|c|c|c|c|c|}
\hline \multirow{2}{*}{$\begin{array}{l}\text { Case } \\
\text { No. }\end{array}$} & \multicolumn{2}{|c|}{ Visual Acuity } & \multirow[b]{2}{*}{ Time Interval (mos) } & \multicolumn{2}{|c|}{$\begin{array}{c}\text { Vol of Optic Nerve } \\
\left(\mathrm{mm}^{3}\right) \text { Covered by } \\
\text { Cumulative Margin Dose }\end{array}$} & \multicolumn{2}{|c|}{$\begin{array}{c}\text { Cumulative Peak } \\
\text { Dose (Gy) }\end{array}$} \\
\hline & Pre-GKS & Last Follow-Up & & 5 Gy & $8 \mathrm{~Gy}$ & Retina & Optic Nerve \\
\hline 1 & 0.2 & 0.2 & 38 & 351.9 & 328.1 & 12.4 & 17.2 \\
\hline 2 & 0.7 & 0.9 & 20 & 18.6 & 5.3 & 11.6 & 14.4 \\
\hline 3 & 0.1 & 0.9 & 20 & 151.7 & 82.9 & 3.6 & 17.2 \\
\hline 4 & 0.01 & 0.02 & 63 & 81.7 & 43.4 & 4.4 & 14.0 \\
\hline 5 & 0.01 & 0.4 & 42 & 168.5 & 123.6 & 16.8 & 16.0 \\
\hline 6 & 0.2 & 0.3 & 58 & 44.1 & 13.9 & 15.2 & 16.8 \\
\hline 7 & 0.05 & 0.5 & 49 & 82.2 & 51.0 & 16.4 & 14.8 \\
\hline 8 & 0.01 & 0.02 & 64 & 127.6 & 88.7 & 0.8 & 20.0 \\
\hline 9 & 0.7 & 0.8 & 40 & 102.1 & 70.3 & 12.4 & 16.8 \\
\hline 10 & 0.02 & 0.15 & 44 & 187.4 & 146.2 & 1.2 & 17.6 \\
\hline 11 & 0.05 & 0.2 & 34 & 211.6 & 211.6 & 21.2 & 17.2 \\
\hline 12 & 0.06 & 0.15 & 74 & 276.6 & 276.6 & 13.6 & 18.0 \\
\hline 13 & 0.9 & 0.6 & 23 & 83.7 & 63.2 & 2.8 & 14.0 \\
\hline 14 & 0.6 & 1.0 & 7 & 212.8 & 145.4 & 12.4 & 18.0 \\
\hline 15 & 0.4 & 0.4 & 20 & 106.2 & 74.5 & 21.6 & 20.8 \\
\hline 16 & 0.15 & 1.0 & 34 & 97.2 & 30.6 & 0.4 & 14.0 \\
\hline 17 & 0.03 & 0.03 & 44 & 155.4 & 113.2 & 4.4 & 17.2 \\
\hline 18 & 0.001 & 0.02 & 36 & 75.6 & 38.5 & 5.2 & 17.2 \\
\hline 19 & 1.0 & 1.0 & 28 & 27.4 & 27.1 & 7.2 & 20.0 \\
\hline 20 & 1.0 & 1.0 & 34 & 165 & 107.6 & 3.2 & 14.0 \\
\hline 21 & 0.01 & 0.03 & 53 & 190.7 & 190.7 & 18 & 20.0 \\
\hline 22 & 0.01 & 0.05 & 37 & 41.8 & 37.1 & 13.6 & 17.6 \\
\hline 23 & 0.01 & 0.02 & 40 & 328.8 & 265.2 & 5.6 & 12.8 \\
\hline
\end{tabular}

the total dose delivered by conventional fractionated radiotherapy. Lesions developing in the orbital region often have pathological characteristics similar to those of intracranial tumors, and their biological responses to single high-dose irradiation are predicted to be similar as well, although there are few reports concerning the application of radiosurgery to orbital lesions. ${ }^{1,8,11,19}$ However, there is an important difference between orbital and intracranial tumors with respect to radiosurgery. In the treatment of orbital tumors, radiation-sensitive structures such as the optic apparatus are inevitably exposed to substantial irradiation in almost all cases, and this may be a major reason for the undervaluation of radiosurgery. Traditional fractionated radiotherapy allows sufficient time for sensitive normal structures to repair and regenerate during the interval between fractions. Fractionated radiosurgery with a Gamma Knife also exploits this advantage of fractionation. We chose 3 fractions for the first patient in our trial, using the most commonly used interval of fractionation, which is 24 hours. A change in fractionation scheme (4 fractions with 12-hour intervals) was implemented in subsequent patients to improve the probability of tumor control by increasing the total dose. Based on previous experience in which hyperfractionated radiation treatment (that is, irradiation twice a day) was used for brainstem gliomas, it was assumed that a 12-hour interval with an increased fraction number would be equally as safe as a 24-hour interval. ${ }^{13}$ At the same time, multisession radiosurgery uses exactly the same dose profile as that used for single-session radiosurgery and minimizes spatial inaccuracy that might result from inconsistent patient setup in each treatment session. In other words, the high conformity and accuracy of multisession radiosurgery allows for a higher dose per fraction, resulting in a higher biologically equivalent dose to the target without increasing the risk of complications in surrounding tissue. In our case series, none of the patients experienced visual deterioration or tumor growth during the follow-up period. These results suggest that multisession GKS may help achieve good tumor control with acceptable risk.

Other authors have published data in a group of patients with cavernous hemangioma treated with GKS. Those authors reported that single-session GKS can significantly reduce tumor size and control the growth of cavernous hemangiomas. ${ }^{10,14}$ Our data showed that fractionated GKS results in comparable tumor control. In our study, cases of cavernous hemangioma showed a significant reduction in tumor volume compared with cases of other types of lesion $(p<0.05)$. This result supports the idea that orbital cavernous hemangiomas are different from cerebral cavernous malformations. Therefore, multisession radiosurgery is an excellent alternative to opera- 
tive intervention and may even replace operative procedures, especially for orbital cavernous hemangiomas.

Despite the low shrinkage rate, multisession GKS is also a good option for the treatment of orbital meningiomas because, even after curative surgery, there is a considerable risk of local relapse. Recurrence rates of up to $15 \%$ for "total" or "radical" resection and more than $75 \%$ for "subtotal removal" have been reported, and additive adjuvant therapy has frequently been used in these cases. ${ }^{4,16}$ Likewise, primary or secondary multisession GKS has been recommended for patients unsuitable for curable resection.

Despite these favorable outcomes, we cannot definitively conclude that fractionated GKS is the treatment of choice for benign orbital tumors. Our series did not have a sufficient number of cases, and long-term follow-up will be necessary to substantiate the potentially curative effects of radiosurgery. Nonetheless, we believe that the present analysis highlights several points. First, fractionated radiosurgery is a well-tolerated surgical procedure that meets most patients' expectations. Second, the rate of tumor volume reduction in cavernous hemangioma is significant and higher than that for other lesions such as meningioma. Third, multisession GKS carries a minimal risk of optic neuropathy with a favorable tumor control rate compared with other treatment modalities such as radiotherapy or open surgery.

\section{Conclusions}

From our experience, 4-session GKS with a margin dose of 4.5-5.5 Gy at the 50\% isodose line in each session can be an alternative option in the treatment of wellcircumscribed benign orbital tumors. Long-term prospective and comparative studies with larger case series are warranted to fully validate the safety and efficacy of this procedure.

\section{Disclosure}

This study was supported by a grant (No. A092255) from the Korea Healthcare Technology R\&D Project, Ministry for Health \& Welfare Affairs, Republic of Korea. The authors report no conflict of interest concerning the materials or methods used in this study or the findings specified in this paper.

Author contributions to the study and manuscript preparation include the following. Conception and design: Lee, Jo, Kong, Seol, Nam. Acquisition of data: Jo, Im, Kim. Analysis and interpretation of data: Lee, Jo, Im. Drafting the article: Jo. Critically revising the article: all authors. Reviewed submitted version of manuscript: all authors. Approved the final version of the manuscript on behalf of all authors: Lee. Statistical analysis: Jo. Administrative/technical/ material support: Im. Study supervision: Lee, Kong, Nam.

\section{References}

1. Adler JR Jr, Gibbs IC, Puataweepong P, Chang SD: Visual field preservation after multisession cyberknife radiosurgery for perioptic lesions. Neurosurgery 62 (Suppl 2):733-743, 2008

2. Arthurs BJ, Lamoreaux WT, Mackay AR, Demakas JJ, Giddings NA, Fairbanks RK, et al: Gamma knife radiosurgery for vestibular schwannomas: tumor control and functional preservation in 70 patients. Am J Clin Oncol 34:265-269, 2011

3. Baumert BG, Villà S, Studer G, Mirimanoff RO, Davis JB, Landau K, et al: Early improvements in vision after fraction- ated stereotactic radiotherapy for primary optic nerve sheath meningioma. Radiother Oncol 72:169-174, 2004

4. Becker G, Jeremic B, Pitz S, Buchgeister M, Wilhelm H, Schiefer U, et al: Stereotactic fractionated radiotherapy in patients with optic nerve sheath meningioma. Int J Radiat Oncol Biol Phys 54:1422-1429, 2002

5. Cantore WA: Neural orbital tumors. Curr Opin Ophthalmol 11:367-371, 2000

6. Dutton JJ: Optic nerve sheath meningiomas. Surv Ophthalmol 37:167-183, 1992

7. Harris GJ: Cavernous hemangioma of the orbital apex: pathogenetic considerations in surgical management. Am J Ophthalmol 150:764-773, 2010

8. Hirschbein MJ, Collins S, Jean WC, Chang SD, Adler JR Jr: Treatment of intraorbital lesions using the Accuray $\mathrm{Cy}$ berKnife system. Orbit 27:97-105, 2008

9. Ito M, Ishizawa A, Miyaoka M, Sato K, Ishii S: Intraorbital meningiomas. Surgical management and role of radiation therapy. Surg Neurol 29:448-453, 1988

10. Khan AA, Niranjan A, Kano H, Kondziolka D, Flickinger JC, Lunsford LD: Stereotactic radiosurgery for cavernous sinus or orbital hemangiomas. Neurosurgery 65:914-918, 2009

11. Kim MS, Park K, Kim JH, Kim YD, Lee JI: Gamma knife radiosurgery for orbital tumors. Clin Neurol Neurosurg 110: 1003-1007, 2008

12. Kondziolka D, Levy EI, Niranjan A, Flickinger JC, Lunsford LD: Long-term outcomes after meningioma radiosurgery: physician and patient perspectives. J Neurosurg 91:44-50, 1999

13. Lewis J, Lucraft H, Gholkar A: UKCCSG study of accelerated radiotherapy for pediatric brain stem gliomas. Int J Radiat Oncol Biol Phys 38:925-929, 1997

14. Liu X, Xu D, Zhang Y, Liu D, Song G: Gamma Knife surgery in patients harboring orbital cavernous hemangiomas that were diagnosed on the basis of imaging findings. Clinical article. J Neurosurg 113 Suppl:39-43, 2010

15. Paek SH, Downes MB, Bednarz G, Keane WM, Werner-Wasik M, Curran WJ Jr, et al: Integration of surgery with fractionated stereotactic radiotherapy for treatment of nonfunctioning pituitary macroadenomas. Int J Radiat Oncol Biol Phys 61: 795-808, 2005

16. Pitz S, Becker G, Schiefer U, Wilhelm H, Jeremic B, Bamberg $\mathrm{M}$, et al: Stereotactic fractionated irradiation of optic nerve sheath meningioma: a new treatment alternative. Br J Ophthalmol 86:1265-1268, 2002

17. Sheehan JP, Kondziolka D, Flickinger J, Lunsford LD: Radiosurgery for residual or recurrent nonfunctioning pituitary adenoma. J Neurosurg 97 (5 Suppl):408-414, 2002

18. Thompson TP, Lunsford LD, Flickinger JC: Radiosurgery for hemangiomas of the cavernous sinus and orbit: technical case report. Neurosurgery 47:778-783, 2000

19. Xu D, Liu D, Zhang Z, Zhang Y, Li Y, Liu X, et al: Gamma Knife surgery in the management of orbital tumors. Clinical article. J Neurosurg 113 Suppl:34-38, 2010

20. Zabramski JM, Kiriş T, Sankhla SK, Cabiol J, Spetzler RF: Orbitozygomatic craniotomy. Technical note. J Neurosurg 89:336-341, 1998

Manuscript submitted April 16, 2012.

Accepted July 24, 2012.

This work was presented in abstract form at the 16th International Meeting of the Leksell Gamma Knife Society, March 25-29, 2012, in Sydney, Australia.

Please include this information when citing this paper: DOI: 10.3171/2012.7.GKS12780.

Address correspondence to: Jung-Il Lee, M.D.,Ph.D., Department of Neurosurgery, Samsung Medical Center, Sungkyunkwan University School of Medicine, 50 Irwon-Dong, Gangnam-gu, Seoul 135-710, Korea. email: jilee@skku.edu. 